\title{
Questua, sacrificio e banchetto rituale nelle feste campestri della Sardegna
}

Begging, sacrifice and ritual banquet in the rural festivals of Sardinia

\section{Sebastiano Mannia}

\section{OpenEdition}

Journals

Edizione digitale

URL: http://journals.openedition.org/aam/336

DOI: $10.4000 /$ aam.336

ISSN: 2038-3215

Editore

Dipartimento Culture e Società - Università di Palermo

\section{Notizia bibliografica digitale}

Sebastiano Mannia, "Questua, sacrificio e banchetto rituale nelle feste campestri della Sardegna ». Archivio antropologico mediterraneo [Online], Anno XXI, n. 20 (1) | 2018, online dal 30 juin 2018, consultato il 27 décembre 2019. URL : http://journals.openedition.org/aam/336 ; DOI : 10.4000/aam. 336

Questo documento è stato generato automaticamente il 27 dicembre 2019.

Archivio antropologico mediterraneo 


\title{
Questua, sacrificio e banchetto rituale nelle feste campestri della Sardegna
}

Begging, sacrifice and ritual banquet in the rural festivals of Sardinia

\author{
Sebastiano Mannia
}

\section{Considerazioni introduttive}

1 In questo lavoro s'intende riflettere sul rapporto che intercorre tra questue, sacrificio e banchetto rituale nelle feste campestri della Sardegna. Nello specifico verranno indagate da un lato le festività in onore della Beata Vergine del Miracolo, di san Nicola da Tolentino e di san Matteo Apostolo che si rinnovano annualmente a Lula nei primi tre fine settimana di settembre, dall'altro il novenario di san Francesco festeggiato dalla comunità di Nuoro dall'1 al 10 maggio e dall'1 al 4 ottobre ${ }^{1}$. Simbolismi, itinera rituali e pratiche devozionali delle feste campestri sono nella maggior parte dei contesti analoghi e pertanto l'analisi di queste festività consente di estendere il quadro interpretativo al più generale complesso di feste campestri della Sardegna e segnatamente a quello delle sue aree più interne (cfr. Angioni 1988, 1991 e 1993; Asole 1985; Gallini 2003; Atzori 1988; Satta 1982 e 1988). I casi considerati sono inoltre rappresentativi di due differenti ordini festivi poiché le occorrenze lulesi si consumano nell'arco di due giorni, mentre nel caso di san Francesco si è di fronte a una festa della durata di nove giorni, i cosiddetti novenari, che prevedono il trasferimento di gruppi familiari più o meno numerosi presso un santuario, e sono da interpretare storicamente come il modello festivo derivante dalle festività campestri della durata limitata di uno o pochi giorni. In tutti i casi si tratta di ricorrenze che si sviluppano tra la primavera e l'autunno, specificamente tra maggio e ottobre, attraverso le quali la «società celebra se stessa, la sua ricchezza, la sua interdipendenza devozionale, la sua appartenenza attraverso l'unicità del culto» (Buttitta 2006: 43). Non c'è probabilmente comunità nell'isola, come ha osservato Giulio Angioni (1988), che non abbia una chiesa 
campestre; sono certamente numerose quelle che annoverano uno o più santuari rurali, «di regola frequentati solo per la festa del santo che ne è titolare, dunque una volta all'anno, non di rado due o anche tre, con iterazioni di ottave» (Angioni 1993: 216). In alcuni casi si tratta di festività gestite da più paesi - è il caso della festa in onore della Madonna di Gonare, condivisa da Sarule e Orani che ad anni alterni si occupano della sua organizzazione; in altri, i santuari si trovano nel territorio di una comunità diversa da quella che organizza i festeggiamenti, come per esempio quello di san Francesco, il novenario di pertinenza dei nuoresi il cui edificio di culto è edificato in agro di Lula. Talora si tratta anche «di santuari che sono stati, in tempi più o meno lontani, chiese di comunità residenti rimaste a testimoniare più $o$ meno vagamente un centro abbandonato dai suoi abitanti trasferitisi in altri centri anche lontani. Questo spiega certi racconti di rivalità tra paesi nel rivendicare un santuario» (Ibidem: 217).

2 Le caratteristiche principali comuni a tutte le feste campestri sono la pratica devozionale e il momento festivo, che si originano, si sviluppano e si consumano all'interno di tre spazi: la chiesa; un ampio cortile adibito a centro comunitario; una serie di piccoli monovani e bivani - cumbessias o muristenes - disposti a cerchio intorno all'edificio di culto o comunque nelle sue vicinanze ${ }^{2}$. Vi sono santuari campestri privi di abitazioni, diffusi in tutta l'isola, mentre nella fascia centrale, ossia dal golfo di Oristano nella parte occidentale a quello di Orosei nel lato orientale, predominano quelli con le dimore annesse. I santuari rimangono chiusi e disabitati per tutto l'anno finché non si rianimano nei giorni dedicati alla festa. Allora gruppi, singoli individui, fedeli, in pellegrinaggio o meno, «verranno, da uno a più paesi, per sciogliere una promessa fatta al Santo e rimanere lì a soggiornare sotto la sua tutela, facendo festa e dandosi ai piaceri dell'ospitalità» (Gallini 2003: 55. Cfr. Moreddu 2008). Le festività campestri sono feste di ringraziamento, di richiesta di grazia, di convivialità sociale; luoghi di preghiera, di pellegrinaggi e processioni verso il santuario, in diversi casi con la scorta dei cavalieri. Tali occorrenze festive si connotano ancora, in parte, per la partecipazione e la collaborazione della comunità: gli individui vi agiscono secondo regole conosciute, interfacciandosi con un comitato organizzatore o con uno o più priori. Fra gli altri elementi in comune, le feste campestri hanno alcuni tratti su cui sostanzialmente si struttura tutto l'impianto festivo e su cui ci soffermeremo: la questua, il sacrificio degli animali offerti, i banchetti rituali.

3 Nel primo paragrafo viene analizzato l'istituto della questua, ossia la raccolta itinerante di beni in natura e denaro, che costituisce uno dei simboli rituali più importanti delle cerimonie festive di area mediterranea. Si delineeranno i diversi tipi di questua che connotano le feste considerate, e in particolare i tempi e gli spazi di raccolta, «rigidamente fissati da tradizioni locali le quali regolano anche altri aspetti connessi a tale importante istituto festivo: i protagonisti e la destinazione finale dei beni raccolti» (Giallombardo 1990: 25).

4 Nel capitolo successivo ci si sofferma sulla complessa istituzione del sacrificio all'interno dell'ordinamento festivo della Sardegna, ovvero sui significati che assume questo fenomeno in una cultura che fonda, soprattutto oggi, sull'offerta di animali e sulla loro uccisione la predisposizione di numerose festività. Infine, ci si concentrerà sui banchetti rituali, nei quali risalta la presenza di cibi a base di carne, in particolare arrosti e bolliti, alternati ad altri alimenti di origine animale, come sanguinacci, cordule, frattaglie. Durante il banchetto si rinnovano relazioni e gerarchie, si esalta l'abbondanza e il consumo, si ribadisce l'appartenenza alla comunità e al culto 
comunitario. Il cibo, in sostanza, diviene un importante e imprescindibile strumento di comunicazione sociale.

\section{Questua}

Uno dei tratti più importanti delle feste campestri è la questua di beni alimentari e denaro, la prima fase fondamentale in cui la comunità in modo più o meno partecipato prende parte al rito festivo.

6 In linea generale le pratiche di questua si sviluppano secondo tempi, modalità e luoghi definiti e sono compiute da soggetti socialmente investiti del ruolo di questuanti e legittimati a svolgerlo: priorati, confraternite, comitati, gruppi di bambini, giovani e adulti. Nella complessità dei riti di questua si possono includere

pratiche che vanno dal furto rituale all'accatto di cibo e/o denaro, in ultima analisi tutte le attività itineranti, connesse a scadenze del calendario festivo, dirette a una raccolta di beni funzionale all'efficacia del rito festivo. Ciò comporta che la questua, laddove prevista, concorre, con il suo di volta in volta specifico articolato simbolico, in maniera decisiva e imprescindibile alla definizione del senso della festa (di “quella" festa) (Buttitta 2016: 159. Cfr., tra gli altri, Buttitta 2006; Clemente 1981, 1982 e 1983; Giallombardo 1990; Grimaldi 1993 e 2012).

7 L'offerta che segue alla richiesta dei questuanti concretizza uno scambio tra beni immateriali - l'auspicio di benessere, salute e prosperità - e beni materiali - alimenti e denaro, posto che in ambito cerimoniale i questuanti rappresentano delle "figure dell'alterità", sono agenti del sacro, e pertanto offrire loro qualcosa rappresenta un investimento utile ai fini dell'ottenimento futuro di un qualche beneficio (cfr. Lanternari 1976; Grimaldi 1993; Buttitta 2006 e 2016). Le offerte, dunque, non vanno ricondotte primariamente alla "dimensione sociale" della questua - i rapporti di reciprocità e redistribuzione che si creano e si rinnovano nelle occasioni festive, espressi massimamente dalla circolazione degli alimenti, evidenziano bene le dinamiche nella e della comunità, che si rifonda, rinsaldando i rapporti familiari, di vicinato e dell'intera collettività - ma si configurano come un dono da rivolgere alle entità trascendenti (Dio, la Madonna, i Santi, i morti) che propiziano il benessere delle persone e, quindi, i cicli della vita individuale e collettiva (in passato anche l'equilibrio e la corretta scansione dei cicli stagionali, l'abbondanza dei raccolti, la prosperità degli armenti). Tali azioni rituali, o comunque diverse loro forme, osserva Ignazio Buttitta, sono

indice della persistenza di un'ideologia magico-religiosa di matrice contadina o comunque, al momento in cui - come spesso oggi accade - i loro sensi e le loro funzioni appaiono slegati da questo più antico orizzonte ideologico e piuttosto riferibili a nuove istanze (riappropriazione della memoria, rafforzamento del senso di appartenenza, riproposizione della tradizione in chiave turistica ecc.), almeno morfologicamente riconducibili a prassi rituali proprie delle società di cultura agropastorale (Buttitta 2016: 162).

Nel caso delle tre festività lulesi, in passato i questuanti, ovvero il comitato organizzatore coadiuvato da amici e parenti, ricevevano in dono lardo, olio, formaggio, vino e soprattutto grano, che veniva venduto e il ricavato era utilizzato per la preparazione dei festeggiamenti. L'offerta del grano non era casuale in una cultura agricolo-pastorale come quella lulese, infatti «sul piano simbolico l'offerta del frumento si riconnette alle arcaiche modalità agrarie dell'offerta primiziale per garantire un 
proficuo svolgersi degli eventi» (Giallombardo 1990: 126). Inoltre, per quanto attiene il significato ideologico, il frumento assolveva «la stessa funzione aggregativa del banchetto e della distribuzione dei pani. Offerto sotto forma di dono esso circolava come segno tangibile della solidarietà comunitaria» (Ibidem: 126). Oggi vengono offerti principalmente soldi e capi di bestiame, con la questua degli animali che ha tempistiche differenti rispetto al giro di raccolta che viene condotto nelle case della comunità. I priori, infatti, in un primo momento contattano tutti i pastori e verificano la loro disponibilità a offrire uno o più capi ovini; in prossimità della festa, coloro che hanno offerto la pecora verranno ricontattati per la conferma della donazione. I pastori, quindi, verranno invitati i giorni antecedenti alla ricorrenza a presenziare e a partecipare alla macellazione dei capi, mentre in seguito riceveranno in contraccambio una bottiglia di vino e un campanaccio, quest'ultimo benedetto dal prete durante la messa della domenica. Le questue che si eseguono nelle case del paese, solitamente in primavera, vengono concordate fra i priorati delle tre feste affinché non si creino sovrapposizioni. I priori, di solito, questuano nelle vie principali mentre i membri del comitato, sas partes, si dividono in gruppi e ognuno questua in un rione del paese. Oltre al denaro ricevono formaggio, pane e più in generale beni alimentari, offerte che vengono contraccambiate con un'immaginetta della Madonna o del Santo.

Il giorno della questua ci ritroviamo di solito nella casa del primo priore. Qui si costituiscono i gruppi, 6-7, e ognuno andrà a questuare in un vicinato. Quando si termina il giro andiamo tutti al santuario dove vengono contati i soldi che ci hanno offerto e mangiamo. Ci sono persone del comitato che preparano da mangiare: antipasti, pasta fatta a mano, fettine, carne in umido o arrosto (dipende dalla carne che è rimasta della festa precedente), patate fritte, verdura, formaggio, seatas, frutta e qualche dolce - di solito qualcuno realizza delle torte (A. P. 1986, intervista del 8.09.2017).

9 Nel caso del novenario di san Francesco, il priore conduce i giri di raccolta in diversi periodi dell'anno. Il primo, in previsione della ricorrenza di ottobre, si svolge di solito a giugno. In base agli impegni del priore e della moglie, accompagnati da parenti, amici e collaboratori, si questua nelle case dei pastori di Nuoro per chiedere il formaggio. A fine settembre si ritirano le pecore offerte per essere macellate e conservate nelle celle frigorifere. Di sera, dopo la questua, i priori offrono sempre la cena ai collaboratori. Per quanto attiene alla ricorrenza di maggio, la consuetudine prevede che la questua possa prendere avvio dal giorno successivo dedicato a sant'Antonio abate, il 17 gennaio; non si può uscire prima. Verso marzo, inizi d'aprile il priore e i suoi collaboratori si recano negli ovili o nei paesi - dove una persona si fa carico di riunire gli animali offerti - per ritirare le pecore o altri beni come formaggio, vino ecc. Per contraccambiare il dono ricevuto, il priore regala ai pastori una bottiglia di vino, un pacchetto di sigarette, una campanella e uno scapolare, sa rezetta, contenente l'immaginetta di san Francesco e una medaglietta; in passato, conteneva anche grano, sale e una moneta. Prima di essere benedetti, il prete apre un sacchettino per controllare il contenuto. In passato lo scapolare veniva inserito all'interno del campanaccio della migliore pecora del gregge per propiziare una buona annata e per preservare gli animali da malattie, furti e incidenti. Gli ovini offerti vanno a costituire un gregge di grandi dimensioni chiamato sas erveches de santu Vrantziscu (le pecore di san Francesco). La prioressa e le collaboratrici, invece, questuano nei paesi di pertinenza del santo, sa vorania. Hanno il vessillo, sa pandela, e una piccola teca contenente la statua di san Francesco, che viene toccata e baciata dai fedeli. L'offerta, solitamente in denaro, viene contraccambiata con 
una immaginetta del santo. La colazione, il pranzo (quando si questua anche di mattina) e la cena sono sempre offerti dai priori.

Per la questua ti organizzi, devi chiedere alle persone chi è disposto a collaborare e ad accompagnarti, se gli fa piacere: las devese prethiare (devi lusingarle). Comunque, coloro che ogni anno aiutano ad organizzare la festa anche se non li cerchi si offrono di aiutarti, perché sono abituati. Noi uscivamo il mercoledì e il venerdì dopo pranzo e il sabato tutto il giorno; partivi la mattina e rientravi di sera. A quel punto offrivamo la cena a queste persone che venivano ad aiutare. Ci sono due, tre amici che si impegnano a preparare da mangiare mentre noi questuiamo (G. P. 1957, intervista del 12.08.2011).

La questua ancora oggi evidenzia bene l'economia delle feste in oggetto, basata su una struttura in cui i rapporti di collaborazione gratuiti, il dono di beni alimentari, di animali, le offerte in denaro sottolineano in una certa misura l'economicamente gratuito e il socialmente vincolante, anche a prescindere dal ruolo che ha assunto l'economia monetarizzata, propria di una società dei consumi che si è andata imponendo a partire dal secondo cinquantennio del secolo scorso e che ha certamente influenzato ma non ancora del tutto trasformato questo importante genere festivo (cfr. Aria 2016). Le feste campestri, pertanto, sussumono tutt'oggi le regole del dare, del ricevere e del ricambiare. Il dono, l'ospitalità, l'obbligo della reciprocità sono norme sociali che mediano i rapporti nella festa e della festa, nei suoi aspetti laici e in quelli più segnatamente religiosi. La reciprocità nello specifico si erge a codice fondamentale della festa: quest'ultima conferma la reciprocità e in essa si struttura. La casa del priore, le cumbessias, i bar, i locali dei banchetti sono spazi in cui l'ospitalità si eleva a rito, con le sue regole e i suoi comportamenti definiti e da definire, e i suoi codici da seguire e rispettare ${ }^{3}$.

11 Le feste in questione e segnatamente il novenario in onore del santo di Assisi continuano dunque a risaltare all'interno del panorama festivo dell'isola e continuano a rappresentare un luogo di fastosità, ostentazione ed esibizione: gli addobbi cerimoniali, la ricchezza di cibi e bevande e il susseguirsi continuo di pranzi e cene, ecc. Oggi più di ieri la vistosità si declina quale tratto fondamentale della festa, grazie anche al generale benessere economico raggiunto nell'isola. I doni, in quanto simboli essenziali e mediatori nella sfera del festivo, sono esibiti e ostentati:

i lunghi tempi delle cerimonie - tempi sottratti alla produzione -, i costi del dono, dell'ospitalità, delle feste affermano un principio a tal punto immediatamente non produttivo, da potersi manifestare nello spreco e, al limite, nella distruzione. La festa valorizza il gratuito, l'effimero: ma in funzione di un obbligo sociale ben preciso, che costituisce il fine verso il quale convergono sforzi e intenzioni di tutti (Gallini 2003: 271).

\section{Sacrificio}

Le motivazioni che i pastori adducono all'offerta delle pecore sono diverse: per devozione, per onorare il Santo, per tradizione, per amicizia e stima nei confronti del priore, per grazia ricevuta, per "fare la festa".

Il sacrificio degli animali, a questo punto, deve essere opportunamente contestualizzato all'interno di una specifica tradizione culturale, che ha assunto la sua dimensione odierna in tempi relativamente recenti. Sino agli anni Cinquanta-Sessanta del secolo scorso, infatti, nemmeno la straordinarietà del momento festivo presupponeva il 
sacrificio di animali nella sua caratterizzazione dispendiosa documentabile oggi. Inoltre, a causa delle ristrettezze economiche, non tutti i capi per la preparazione della festa venivano donati; la maggior parte veniva acquistata. Così riferisce un informatore:

Prima ammazzare le pecore era un lusso. A su Meraculu [festa della Madonna del Miracolo] si andava la sera, si ammazzavano cinque capre, si preparavano frattaglie e interiora e finiva lì. L'indomani si faceva il brodo e si mangiava questo con la carne lessa. Hanno iniziato a fare la festa, pressappoco come è oggi, dopo gli anni Sessanta (G. P. 1941, intervista del 13.08.2011).

È noto che nelle società pastorali l'uccisione di animali allevati rappresentava un atto decisamente critico e traumatico. Marcello Massenzio, in linea con Vittorio Lanternari (1976), ha ben chiarito che «l'avversione ad uccidere gli animali allevati rende necessario il ricorso a procedure rituali riconducibili, in astratto", a specifiche direttive, tra cui: «proiettare l'azione dell'uccidere nella dimensione della festa che, essendo fuori dalla norma, può accogliere in sé atti analogamente fuori dall'ordinario»" (Massenzio 1994: 85). Il sacrificio è possibile perché è rito, perché è regolato dalla tradizione: "soltanto grazie alle mediazioni culturali garantite dalla prassi rituale si apre la strada, normalmente preclusa, che porta alla messa a morte delle bestie allevate, nonché al consumo alimentare delle loro carni» (Ibidem: 88$)^{4}$.

Come si inquadra, dunque, il sacrificio degli animali all'interno delle dinamiche culturali e religiose nei contesti etnografici considerati e qual è il senso che assume, soprattutto in relazione ai cambiamenti delle strutture socio-economiche tradizionali ? Seguendo Maurice Bloch (2005), e cioè evitando analisi di carattere generale e transculturale, ci si soffermerà sulle pratiche di sacrificio nell'ambito delle realtà lulese e nuorese, luoghi e tempi particolari, riprendendo le coordinate analitiche, a mio avviso utili, ancora valide e dense di significato, proposte da Valerio Valeri $(1994,1985)$, che del sacrificio individua quattro momenti: il reperimento dei beni da distruggere (induzione); la loro distruzione (presa della vita); la separazione, in parte o in tutto, dai beni distrutti, che verranno destinati alle divinità (rinuncia); il consumo dei beni distrutti da parte di coloro che partecipano al rituale. Quest'ultimo momento è la parte più sociale e festiva del rito, e in diversi casi, per Valeri, l'apice del sacrificio. Secondo l'antropologo non è necessario che siano sempre presenti tutte le dimensioni, che possono

essere variamente modulate, ma anche totalmente assenti. Infine, sebbene la presa della vita, o distruzione della cosa, sia presente in ogni atto sacrificale (in senso sia reale sia simbolico, sia sotto forma di azione sia sotto forma di evocazione), non è affatto detto che ogni soppressione della vita o distruzione di qualcosa sia da considerarsi di per sé un sacrificio, ma va considerata tale solo se entra in relazione con almeno uno degli altri aspetti (Fabietti 2014: 127).

Ognuno di questi implica sempre tre relazioni: tra esseri umani ed entità trascendenti; tra esseri umani e beni sacrificati; tra esseri umani che consumano il sacrificio o vi prendono comunque parte (Ibidem: 131). La seconda relazione si conclude quasi sempre con il pasto comune ${ }^{5}$. Nei casi etnografici considerati in questa sede, le tre relazioni sono evidentemente presenti ed emergono in modo determinante, posto che la macellazione degli animali è centrale nel ragionamento che si conduce anche perché ha importanti valenze sociali e religiose ${ }^{6}$. In linea con Bloch (2005) il termine sacrificio non è che un modo per designare un insieme di elementi proprio di un gruppo più ampio di rituali, non vi è dunque ragione di limitare l'analisi all'uccisione e al consumo, ignorando le altri fasi, anche perché non c'è una distinzione, non vi sono fasi temporalmente e spazialmente scisse. Il tutto va analizzato sul piano rituale come una 
totalità. Sono probabilmente questi vari livelli che hanno permesso al sacrificio di perpetuarsi nel tempo. Per Cristiano Grottanelli,

le due principali trasformazioni che consentirono la sopravvivenza dell'immolazione di vittime animali riguardano entrambe la destinazione della vittima nell'ambito dello scambio sacrificale. Prima di tutto, la vittima non poteva essere offerta al dio unico dei cristiani, che, trascendente e onnipotente, non ne aveva bisogno: dovendola dunque strappare ai demoni, era possibile immolarla cioè ucciderla sacralmente - destinandola al consumo umano durante una festa della "vera religione". In secondo luogo, le parti riservate a una sfera "diversa" erano destinate ai poveri - magari ai pellegrini poveri, convenuti per la festa (Grottanelli 1999: 83).

È questo il caso di numerose occorrenze cerimoniali - comprese quelle qui indagate dove una parte della carne viene tutt'oggi offerta alle persone meno abbienti, agli anziani e ai malati che non possono partecipare al banchetto festivo. Per la Madonna del Miracolo - festività istituita nel 1899 - l'atto costitutivo chiariva che la festa fosse predisposta in particolare per «sollevare i poveri di Lula e specialmente gli orfani, mediante sussidi da distribuirsi dai fondatori in occasione della festa». I poveri e gli orfani sono coloro che: «paradigmaticamente esprimono tutte le possibili minacce connesse alla mancanza di status sociale e quindi umano» (Giallombardo 1990: 119). Fino ad un recente passato, coinvolgerli nel pasto festivo

assolveva alla funzione di riscattare ritualmente le discriminazioni esistenti all'interno della comunità per ripristinare il tempo mitico dell'eguaglianza e della coesione sociale, messe in crisi nel tempo profano. Oggi il pranzo ai poveri si manifesta come istituto culturalmente adeguato a esorcizzare attraverso la rifondazione mitica dell'unità del gruppo, esso stesso ormai "mitico", la crisi dello sradicamento sociale e culturale incombente sugli individui (Ibidem: 119).

Nel caso delle feste lulesi, è con la locuzione a sas pethas (lett. "alle carni") che si indica il giorno deputato al sacrificio degli animali. A faccher sas pethas (lett. "a fare le carni") invece indica proprio l'atto. Chiunque può partecipare alla macellazione dei capi e chiunque può prendere parte al pranzo che si terrà dopo la macellazione degli animali. Tuttavia, solo i pastori-offerenti, coloro che vengono invitati formalmente o fanno parte del comitato organizzatore vi prenderanno parte. È questo uno dei vari livelli di strutturazione gerarchica che si va a comporre all'interno dell'impianto festivo generale. Il giovedì e il venerdì, dunque, i pastori portano gli ovini offerti ai santuari. Il giovedì vengono macellati pochi capi, circa una decina, per preparare i sanguinacci e la carne per il pranzo del giorno successivo, quando verranno sacrificati tutti gli animali donati. Il venerdì, infatti, è una sorta di pre-festa per i pastori: il priorato e il comitato organizzatore invitano appositamente gli allevatori a portare le pecore in questa giornata in maniera tale che si vedano "costretti" a presenziare al banchetto che viene organizzato per loro, ma anche per amici e parenti. Negli ultimi anni vengono macellate anche 50-60 pecore. Un'informatrice mi ha riferito:

Ancor più importante della festa è il pranzo di ringraziamento che si organizza per $i$ pastori; è la festa dei pastori. Si cucinano allora pasta fatta a mano, fettine impanate, cordula, cervella, seatas, ecc. Per quel che mi riguarda, se il giorno in cui si sacrificano gli animali non vengono i pastori da me invitati, lo considero come che la festa sia andata male (R. P. 1984, intervista del 8.09.2017).

19 Più ampia la dimensione sacrificale nel caso di san Francesco, dove vengono offerte anche 500 pecore, la maggior parte delle quali viene macellata per le esigenze della festa nei giorni immediatamente precedenti. 

alcuni casi questa fase viene compiuta all'aperto - sempre all'interno dell'area santuariale. Agli ovini, dopo esser stati legati e appesi per le zampe posteriori, viene recisa la carotide - la consuetudine prevede che gli animali vengano uccisi riducendo al massimo la "crudeltà". Tale atto richiede una certa manualità e abilità e infatti l'avvio delle operazioni coincide con una suddivisione automatica dei ruoli: alcuni uccidono gli animali, altri raccolgono il sangue, altri li squartano, altri puliscono le interiora, altri depositano i capi macellati in celle frigorifere. Una manualità acquisita, un saper fare consolidato e una tradizione gastronomica pressoché invariata sono alla base di questo atto. Una procedura che prevede pratiche, gesti, simbolismi e regole definiti. Le persone più abili sono così ricercate e la prestazione d'opera è gratuita: rientra nei circuiti di scambio e di amicizia propri delle relazioni comunitarie che in questo senso rivestono ancora una certa importanza soprattutto in ambito festivo. Così un informatore:

La prima cosa che devi fare nelle nostre feste è quella di cercare gli amici e i parenti che sai che ti potranno aiutare. Senza di loro la festa non la puoi fare. I collaboratori li devi contattare non 15 giorni prima della festa, devi cercarli in tempo e chiedere se hanno piacere di aiutarti. Ci sono passaggi a cui devi stare attento, perché è fondamentale (A. M. 1957, intervista del 12.08.2011).

L'uccisione dell'animale, pertanto, ha una grande rilevanza sociale, poiché «i fatti e i gesti che mostra mettono in evidenza i principi essenziali della vita collettiva: principio dello scambio e della provvista, principio della condivisione e del reciproco aiuto, principio della divisione sessuale del lavoro; la sua cucina mette in evidenza le categorie culinarie essenziali, così come quelle del gusto e della sensibilità alimentare» (Verdier 1990: 357, cit. in Faeta 2012: 224). Quando le operazioni di macellazione volgono al termine, infatti, ci si riunisce per pranzare. Si mangia pasta, carne di pecora in umido, talora fettine. È questo uno dei tanti momenti in cui si aderisce al momento festivo, sottolineato in primis, ma non esclusivamente, dall'abbondanza alimentare.

\section{Banchetto rituale}

Questua e sacrificio sono, quindi, le più importanti azioni rituali che introducono la parte più rappresentativa e partecipata delle feste campestri: il banchetto. Il cibo viene distribuito e la collettività si ritrova a condividere il pasto comune, in comune. La dimensione del cucinare e del mangiare, legata al sacrificio, che in primis RobertsonSmith (1894) aveva rintracciato e Detienne e Vernant (1982) tra gli altri hanno ribadito, assume un importanza decisiva. In questa direzione, assumiamo quanto ha osservato Burkert, ossia che l'uccisione degli animali, ovvero «l'esperienza della morte, organizzata dall'atto di violenza dell'uomo in forza di una necessità già data», è congiunta all'aspetto umano, «troppo umano: il mangiare, il festoso banchetto sacrificale di quanti per esso e a opera di esso vengono sacralizzati» (1981: 28).

Tutto il periodo festivo è contrassegnato dagli eccessi alimentari, dall'ostentazione del cibo, dalle tavole continuamente apparecchiate e strabordanti di carne. La dimensione del mangiare nelle feste campestri isolane rimane pertanto centrale e la relazione con la carne è ribadita in tutte le fasi che precedono, seguono e posticipano l'occorrenza festiva. L'elemento carneo connota la festa, la sua riuscita, la sua memorabilità. Il piano alimentare assume un'importanza fondamentale poiché è attorno al cibo che si 
instaurano, si consolidano e si rinnovano i rapporti sociali (cfr. Douglas 1985 e 1987). C'è una nota di fondo, come ha sottolineato di recente Paolo Apolito, che accomuna le feste, ossia il gusto e la necessità «di relazione, dunque di condivisione, dunque di riconoscimento di umanità comune» (Apolito 2014: 194) ${ }^{7}$. Nel caso delle occorrenze lulesi i banchetti si limitano alla cena del sabato, dove vengono serviti brodo di carne, lesso, carne in umido, cordula, sanguinaccio, trippa e formaggio, e al pranzo della domenica che ripropone quasi invariato il menù della sera precedente, ad eccezione del banchetto per san Nicola dove il brodo viene sostituito dalla pasta. Nel caso dei novenari, invece, le tavole vengono apparecchiate mattina e sera per nove giorni e la festa di san Francesco, come già documentò Clara Gallini negli anni Sessanta, sussume ancora oggi più di altre, nel panorama festivo della Sardegna, i tratti della straordinarietà. La festa si struttura sulla fastosità, sugli eccessi, soprattutto alimentari, sul prestigio e sull'onorabilità di cui gode il priore all'interno della comunità, di cui sarà valutata l'ospitalità, la magnificenza con cui allestirà $\mathrm{i}$ banchetti, le modalità con cui accoglie $\mathrm{i}$ fedeli e i visitatori che accorrono al santuario.

La festa dipende da chi sono i priori, da come si presentano. Devono saper trattare bene le persone, in particolare i pellegrini, i cavalieri e i novenanti; sono loro che "fanno" la festa. Le attenzioni principali devono essere rivolte a loro, perché sono $\mathrm{i}$ protagonisti più importanti della festa di san Francesco (P. S. 1931, intervista dell'8.08.2011).

La carne ha in questo contesto un ruolo centrale e fra i destinatari privilegiati si inscrivono i novenanti che per nove giorni occupano sas cumbessias. Prima dell'inizio della novena, il priore e i collaboratori macellano un certo numero di capi ovini per distribuire la carne alle famiglie insediate nel santuario, a giorni alterni e per almeno tre o quattro volte durante tutto il periodo del novenario. In passato era consuetudine offrire un giorno sos pinzos, sanguinaccio, cordula, frattaglie, ed un giorno la carne. Oltre a questa distribuzione rituale e oltre ai pranzi e alle cene offerti ai visitatori e ai fedeli che si recano quotidianamente alla festa - a base di pecora bollita con patate, carne in umido, sanguinaccio e su filindeu, una pasta tipica di Nuoro cucinata nel brodo di pecora e condita con formaggio - diversi sono i banchetti che hanno per protagonisti i priori. Per esempio l'8 maggio, dopo l'arrivo al santuario dei cavalieri e del nuovo priore, e dopo aver compiuto i giri rituali intorno alla chiesa, i cavalieri stessi vengono invitati dal vecchio priore a consumare una cena a base di arrosti di agnello e di maialetto. Tradizionalmente la cena deve essere preparata con i beni alimentari scambiati dai priori: il giorno 7 quello in carica dona al nuovo priore 7 pecore; per sua parte quest'ultimo, l'8 mattina, contraccambia con un maialetto, due agnelli, il vino bianco, l'olio, il pane, la frutta. Al priore uscente spetta, inoltre, organizzare il pranzo del 9; la cena, invece, verrà predisposta dal priore entrante, dopo aver chiesto la sera dell's la disponibilità dei cuochi e degli aiutanti. Il priore e la prioressa nuovi devono prethiare sas pessones, lusingare i collaboratori, per l'aiuto annuale. Tutta l'organizzazione festiva è fondata su un circuito di scambio e reciprocità con regole tacite che devono essere opportunamente seguite. Il 9 sera, dunque, si consuma una sorta di passaggio simbolico dal vecchio al nuovo priore dei locali, della cucina e del salone dove si mangia. Alla cena prendono parte i priori uscenti, i cavalieri, i parenti, gli amici e i collaboratori che vengono serviti dalla "nuova organizzazione". Se il priorato entrante è ben accetto, si comporta e si relaziona adeguatamente i cavalieri romperanno pochi piatti - due, tre in segno di buon augurio; al contrario, se il priore tiene un comportamento riprovevole, non presta la giusta attenzione a certe dinamiche relazionali, non si mostra ospitale, 
potranno essere rotti numerosi piatti in segno di disapprovazione. Un altro momento importante si sviluppa la mattina del 10: i novenanti lasciano sas cumbessias, i priori e i cavalieri fanno tre giri intorno alla chiesa, controllano che il santuario sia vuoto e in ordine, chiudono i cancelli d'ingresso e partono a cavallo verso Marreri, una località distante circa $10 \mathrm{~km}$ da Nuoro. Qui il parroco di Lula impartisce una benedizione ai presenti dopodiché tutti i convenuti consumano il pranzo organizzato dai nuovi priori, s'arbore, a base di porcetto, pecora e formaggio arrosto. Tale banchetto costituisce il biglietto da visita del nuovo priorato, che recupererà le spese sostenute per la sua realizzazione nel corso delle questue annuali. Dopo il pranzo, il priore entrante, munito di una bisaccia, accompagnato dal figlio che reca la teca con il santo e dai priori vecchi, questua tra le persone affluite al convito. Sino agli anni Cinquanta del secolo scorso, a s'arbore partecipavano il priore vecchio, quello nuovo e i collaboratori, mentre i cavalieri acchian s'arbore a banna, ossia pranzavano in uno spazio adiacente a quello dei priori, consumando i beni alimentari trasportati l's: ciascuno contribuiva, solitamente, con uova bollite, vino e un agnello, che venivano sacrificati per l'occasione ${ }^{8}$.

\section{Considerazioni conclusive}

Le feste campestri per uno, due o più giorni registrano la partecipazione di centinaia di persone della comunità ed estranei invitati da membri della comunità: la preghiera, il divertimento, il "rispetto della tradizione", il mangiare, ecc. sono interpolati, costruendo differenti livelli di senso fra uomini e santi, fra uomini e animali, fra uomini e uomini, fra vivi e morti. Sono feste che costituiscono l'avvenimento più atteso per numerosi paesi, l'evento più importante dell'anno, e un motivo di grande prestigio soprattutto per i priori. Osserva Francesco Faeta:

Gruppi di famiglie, con la reciprocità instaurata attraverso i banchetti, si riconoscono come alleate, come membri di un segmento comunitario e, insieme, come membri di una comunità. Ciascuno, inoltre, familiarizza con una condizione in cui si dona senza ricevere nulla in cambio; ciascuno sperimenta come il donare crei considerazione e prestigio. Il banchetto è, inoltre, occasione privilegiata per verificare la rete sociale, in rapporto con l'intera comunità: nel memorizzare il complicato intreccio degli inviti; nel tener conto [degli animali] già uccisi e di quelli ancora da uccidere, delle pantagrueliche mangiate e bevute, dei doni fatti e ricevuti; nel discutere, nominare, verificare interdetti ed esclusioni, la rete è mostrata a se stessi e alla comunità (Faeta 2012: 221-222).

Nel momento festivo, attraverso il cibo, la condivisione del cibo, si ricompatta e si rinnova quindi l'ordine sociale oltreché il culto religioso. Solo apparentemente, però, si crea coesione e si annullano le gerarchie. Le differenze sociali non si affievoliscono ma si evidenziano e si rimarcano. Ad esempio, se i priori non sono integrati perfettamente nel tessuto e nella vita comunitaria, tutta la festa ne risente in termini di partecipazione e di offerte. C'è un giudizio e un controllo sociale nella e della festa. E non è solo il priore in questo senso a subire il giudizio ma più estesamente il suo gruppo parentale. La perenne dimensione di conflittualità - talora in modo sarcastico, altre serio - dei componenti dei comitati organizzatori delle diverse feste, si avvia già con la questua. La festa di quel dato priorato verrà ricordata per il numero di persone che vi hanno preso parte, per il grado di ospitalità, per la bontà e l'abbondanza dei cibi offerti. Si fanno i raffronti, si ricorda il banchetto dell'anno precedente, si stabiliscono differenze e gerarchie?. 
La questua, il sacrificio, il consumo rituale degli animali macellati sono istituti culturali che hanno subito evidenti trasformazioni nel tempo a seguito di cambiamenti di diverso ordine, e tuttavia nelle modalità con cui sono stati descritti permangono assolutamente vitali. Anche l'immolazione di animali è ancora possibile - e praticata al di là della sfera legale che proibisce sostanzialmente la macellazione clandestina e non la giustifica in ottica festiva -, se non altro per la destinazione della carne al consumo umano in onore di un santo o della Madonna. In base a quanto osservato, pertanto, questua, sacrificio e condivisione del cibo nei banchetti rituali definiscono da un lato il culto religioso, dall'altro, specificamente, l'ordine della società. In linea con Walter Burkert: «Nel momento in cui la morte diventa uccisione e il partecipante uccisore, la morte stessa è diventata opera della propria volontà, fattibile, ripetibile e proprio per questo superabile nel pasto solenne, durante il quale si convalida la volontà di vivere di chi sopravvive» (1981: 53).

\section{BIBLIOGRAFIA}

Angioni G., 1988 «Santuari e sagre di campagna», in G. Angioni, A. Sanna (a cura di), L'architettura popolare in Italia - Sardegna, Laterza, Roma-Bari: 120-128.

Angioni G., 1993 «Sagre e santuari della montagna», in I. Camarda (a cura di), Montagne di Sardegna, Carlo Delfino, Sassari: 215-223.

Angioni G., 1991 «Feste e santuari campestri», in A. Oppo (a cura di), La provincia di Oristano. Il lavoro e la vita sociale, A. Pizzi, Cinisello Balsamo: 177-188.

Apolito P., 2014 Ritmi di festa. Corpo, danza, socialità, il Mulino, Bologna.

Aria M., 2016 I doni di Mauss. Percorsi di antropologia economica, Cisu, Roma.

Asole A., 1985 «Attorno ai santuari campestri le "cumbessìas" dei novenanti», in Sardegna: l'uomo e le montagne, Silvana Editoriale, Milano: 169-176.

Atzori M., 1988 Cavalli e feste. Tradizioni equestri della Sardegna, L'Asfodelo, Sassari.

Bloch M., 2005 Da preda a cacciatore. La politica dell'esperienza religiosa, Raffaello Cortina, Milano.

Brelich A., 2003 Introduzione alla storia delle religioni, Edizioni dell'Ateneo, Roma.

Burkert W., 1981 Homo necans. Antropologia del sacrificio cruento nella Grecia antica, Boringhieri, Torino.

Buttitta I. E., 2006 I morti e il grano. Tempi del lavoro e ritmi della festa, Meltemi, Roma.

Buttitta I. E., 2016 «Il destino nelle parole. Questue, mascheramenti e formule augurali», in P. Sisto, P. Totaro (a cura di), Maschera e linguaggi, Progedit, Bari: 159-225.

Calame C., 1981 «Presentazione» a W. Burkert, Homo necans. Antropologia del sacrificio cruento nella Grecia antica, Boringhieri, Torino: 7-14.

Clemente P., 1981 «Maggiolata e Sega-la-vecchia nel senese e nel grossetano. Note sulla festa», in C. Bianco, M. del Ninno (a cura di), Festa. Antropologia e semiotica, Nuova Guaraldi, Firenze: 46-57. 
Clemente P., 1982 «I canti di questua: riflessioni su una esperienza Toscana», in La ricerca folklorica, 6: 101-105.

Clemente P., 1983 «La circolazione di uomini, attività, beni nei "canti di questua. Riflessioni teorico-metodologiche», in M. Fresta (a cura di), Vecchie segate ed alberi di maggio: percorsi nel teatro popolare toscano, Ed. del Grifo, Montepulciano: 125-158.

Del Piano M., 1972 La Sagra di San Francesco di Lula, Ettore Gasperini Editore, Cagliari.

Deledda G., 1895 «Tradizioni popolari di Nuoro (Sardegna)», in Rivista delle tradizioni popolari italiane, a. II, Fasc. VI: 401-450.

Deledda G., 1900 «Elias Portolu», in Nuova Antologia, voll. LXXXVIII-LXXXIX, fasc. 687-691.

Deledda G., 1995 «San Francesco», in Ead., Sangue sardo, Tascabili Economici Newton, Roma: 73-87. Detienne M., Vernant J.-P., 1982 La cucina del sacrificio in terra greca, Boringhieri, Torino.

Douglas M., 1985 Antropologia e simbolismo. Religione, cibo e denaro nella vita sociale, il Mulino, Bologna.

Douglas M. (ed.), 1987 Constructive Drinking: Perspectives on Drink from Anthropology, Cambridge University Press, Cambridge.

Fabietti U., 2014 Materia sacra. Corpi, oggetti, immagini, feticci nella pratica religiosa, Raffaello Cortina, Milano.

Faeta F., 2012 «I maiali di Re Carnevale. Una mnemotecnica», in P. Sisto, P. Totaro (a cura di), La maschera e il corpo, Progedit, Bari: 212-227.

Gallini C., 2003 Il consumo del sacro. Feste lunghe di Sardegna, Ilisso, Nuoro.

Giallombardo F., 1990 Festa orgia società, Flaccovio, Palermo.

Girard R., 1980 La violenza e il sacro, Adelphi, Milano.

Grimaldi P., 1993 Il calendario rituale contadino. Il tempo della festa e del lavoro fra tradizione e complessità sociale, Franco Angeli, Milano.

Grimaldi P., 2012 Cibo e rito. Il gesto e la parola nell'alimentazione tradizionale, Sellerio, Palermo.

Grottanelli C., 1999 Il sacrificio, Laterza, Roma-Bari.

Grottanelli C., Parise N. F., Solinas P. G. (a cura di), 1984 «Sacrificio, organizzazione del cosmo, dinamica sociale», in Studi Storici - numero monografico, a. 25, n. 4.

Grottanelli C., Parise N. F., 1993 Sacrificio e società nel mondo antico, Laterza, Roma-Bari.

Hamerton-Kelly R. G. (ed.), 1987 Violent Origins, Stanford University Press, Stanford.

Hubert H., Mauss M., 2002 Saggio sul sacrificio, Morcelliana, Brescia.

King M., 1992 «Sulle tracce della tradizione. San Francesco di Lula», in Sardegna antica, 2: 26-29.

Kirova T., Tramontin A., Bergamini A., 1984 «Architetture della religiosità popolare nella Sardegna del XVII secolo: "cumbessias" e "muristenes"», in T. Kirova (a cura di), Arte e cultura del '600 e del '700 in Sardegna, Edizioni scientifiche italiane, Napoli: 267-280.

Lanternari V., 1976 La grande festa. Vita rituale e sistemi di produzione nelle società tradizionali, Dedalo, Bari.

Lincoln B., 1991 Death, War, and Sacrifice. Studies in Ideology and Practice, The University of Chicago Press, Chicago. 
Mannia S., 2014 «Il consumo del sacro. Feste lunghe di Sardegna quarant'anni dopo», in A. Musco, G. Parrino (a cura di), Santi, santuari, pellegrinaggi, Officina di Studi Medievali, Palermo: 165-182.

Mannia S., 2017 «La fiera dell'ospitalità e delle vanità. La festa novenaria di san Francesco di Lula», in Il Folklore d'Italia, 3: 36-41.

Massenzio M., 1994 Sacro e identità etnica. Senso del mondo e linea di confine, Franco Angeli, Milano.

Miller D., 1998 Teoria dello shopping, Editori Riuniti, Roma.

Moreddu E., 2008 In prossimità dei luoghi. Villaggi di cumbessias, gioco dell'arte e un modo particolare dell'indugiare, Franco Angeli, Milano.

Mori A., 1952 «Centri religiosi temporanei e loro evoluzione in Sardegna», in Studi Sardi, X-XI: $389-402$.

Mossa V., 1950 «Le "cumbessias” o "muristenes"», in Ichnusa, 2: 55-71.

1953 Architettura religiosa minore in Sardegna, Gallizzi, Sassari.

Smith W. R., 1894 Lectures on the Religion of Semites, Adam \& Charles Black, London.

Saba I., 1975 «I novenari e la festa di S. Francesco di Lula», in La Musica Popolare, 2: 30-33.

Satta M. M., 1982 Riso e pianto nella cultura popolare. Feste e tradizioni sarde, L'Asfodelo, Sassari.

Satta M. M., 1988 «Le feste campestri», in M. Brigaglia (a cura di), Le chiese nel verde. Architetture religiose rurali nella provincia di Sassari, Silvana editoriale, Milano: 145-155.

Valeri V., 1985 Kingship and Sacrifice. Ritual and Society in Ancient Hawaii, The University of Chicago Press, Chicago.

Valeri V., 1994 «Wild Victims: Hunting as Sacrifice and Sacrifice as Hunting in Huaulu», in History of Religions, vol. 34, n. 2: 101-131.

Verdier Y., 1990 «Le langage du cochon», in T. Jolas et al., Une campagne voisine. Minot, un village bourguignon, Éditions de la Maison des sciences de l'homme, Paris: 357-375.

\section{NOTE}

1. I dati che confluiscono all'interno di questo contributo sono l'esito di rilevamenti diretti, interviste strutturate, conversazioni informali di una più ampia ricerca che da diversi anni si conduce su alcune festività campestri della Sardegna alla luce dei processi di continuità e di cambiamento che le hanno interessate. Nel caso specifico delle feste lulesi non esiste una letteratura al riguardo mentre per quanto attiene al novenario di san Francesco, oltre al lavoro di Clara Gallini (2003) e ai resoconti di Grazia Deledda (1895, 1900 e 1995), si rimanda a Del Piano 1972; Saba 1975; Satta 1982; Atzori 1988; King 1992; Mannia 2014 e 2017. Si vedano inoltre i documentari, entrambi intitolati San Francesco di Lula, di Paolo Sanna (1984, produzione RAI) e di Fiorenzo Serra (1986, produzione ISRE). Di Serra si veda, inoltre, il documentario La novena (1967), http://www.sardegnadigitallibrary.it/index.php?xsl=626\&id=191044.

2. Sull'architettura dei santuari campestri si veda: Mossa 1950 e 1953; Mori 1952; Kirova et al. 1984; Angioni 1988.

3. Come ha osservato Matteo Aria, il paradigma antropologico della reciprocità e dello scambio è stato oggetto di ripensamento critico a partire dagli anni Ottanta del secolo scorso. La contemporaneità capitalistica, ovvero l'epoca e la società dei consumi, ha indubitabilmente influenzato il dibattito così come gli stessi meccanismi di reciprocità sottesi all'istituto del dono, e tuttavia, per riprendere le parole dello stesso Aria, «lo stato e il mercato ridefiniscono gli spazi 
e i tempi dello spirito del dono senza per questo soffocarli; di conseguenza lo sguardo antropologico dovrebbe soffermarsi ad indagare le pratiche di dono intese come scarti lasciati liberi dai grandi meccanismi della politica e dell'economia, o come "momenti dove si rendono visibili le articolazioni tra le soggettività astratte che si muovono nel mercato e nello stato e quelle concrete che stanno al centro dei legami locali e personali"» (Aria 2016: 58-59). Dono e mercato sono intrecciati e convergono nella dimensione della festa. Nelle ultime pagine del suo lavoro, Aria si sofferma brevemente sulla "condivisione festiva", portando a esempio i novenari sardi studiati da Clara Gallini negli anni Sessanta-Settanta del Novecento, dei quali risalta «una tipica economia del dono - quella agropastorale familiare della provincia di Nuoro - fondata sulla reciprocità e sulla norma espressa dall'adagio "oggi a me, domani a te"» (Aria 2016: 153). I novenari sardi continuano a essere spazi festivi di condivisione, compartecipazione, convivialità, anche grazie al dono, che assume inoltre un ruolo decisivo nella costruzione del prestigio e dei rapporti di potere. Le performance festive, d'altronde, «certo sono azioni estetiche, ma sono anche sempre azioni politiche e sociali. Perché allo stesso modo in cui si fa la società e la si trasforma nelle ovvie e quotidiane azioni politiche e sociali, si fa società e la si trasforma festeggiando» (Apolito 2014: 235).

4. Sull'istituto del sacrificio riflettono da tempo storici delle religioni e antropologi. Per approfondimenti, oltre ai riferimenti nel testo, rimando tra gli altri a: Brelich 2003; Girard 1980; Grottanelli, Parise 1993; Grottanelli et al. 1984; Hamerton-Kelly 1987; Hubert, Mauss 2002; Lincoln 1991. Le teorie sul "sacrificio" e sul "consumo" elaborate da alcuni autori, per esempio Hubert e Mauss e Bataille, sono riprese da Daniel Miller in uno studio dove propone l'analogia tra il sacrificio e lo shopping. Quest'ultimo, secondo l'antropologo, può anche essere una pratica rituale: "Alla base vi è una logica di sacrificio il cui obiettivo è quello di creare soggetti che desiderano, che si aspettano qualcosa. Il sacrificio si basava su riti intesi a trasformare la consumazione in devozione. Lo shopping inizia con un rito dello stesso genere, che nega la mera consumazione in omaggio ai più elevati fini della parsimonia. E termina come impegno a costituire sia la immediatezza che le dinamiche di determinate relazioni di amore» (Miller 1998: 200).

5. Grottanelli ha osservato: «l'uccisione dell'animale commestibile e il consumo del cibo carneo coincidono - quando non con la caccia - con la pratica sacrificale» (1999: 41).

6. Sul piano rituale, osserva Calame, il sacrificio «consente la comunicazione tra gli uomini e gli dei. [...] Il sacrificio realmente eseguito [...] è un mezzo per far partecipare gli dei, attraverso l'intermediario del contatto istituito dal dono di un animale, alla sequenza di atti rituali costituenti la festa; il sacrificio rituale non è che un elemento di questa sequenza e vi si colloca per conferire all'insieme di questi atti un valore religioso» (Calame 1981: 13).

7. Continua Apolito: «Facendo performance di festa - soprattutto ma non solo quella istituzionale - si fa la società. Per questo la festa non è mai una forma data, per quanto possa essere istituzionalizzata. È sempre un evento. Cioè un'occasione in cui si mettono in gioco le relazioni tra le persone e si rischia che quelle relazioni mutino, si consolidino o si rompano. Non è uno specchio della società, del gruppo, ma un suo movimento. In esso la società trascorre, passa, viene a trovarsi in un altro punto. Vive e attraversa la sua congiuntura storica. Le sfide, i conflitti, le competizioni tra performer non riflettono statisticamente le articolazioni interne della società in festa, le sue divisioni strutturali, ma sono un modo in cui queste vengono vissute e trasformate» (Apolito 2014: 236. Cfr. pp. 234-235 ss.).

8. Anche la ricorrenza di ottobre, dall' 1 al 4 , è un susseguirsi di inviti che prevedono, secondo la consuetudine, caffè, vov e liquore di mirto, amaretti e biscotti, ma soprattutto su filindeu, sanguinaccio, cordula, pecora bollita e patate.

9. A proposito di queste dinamiche Paolo Apolito ha scritto: «La competizione e il conflitto in una festa sono il frutto della sua stessa dinamica, che è sempre sfida tra performer. In tutto il mondo abbondano feste in cui le sfide tra performer sono istituzionalizzate. Sfide cioè non casuali o 
legate alle singole circostanze e singole persone, ma previste, l'oggetto stesso della festa. Talvolta non sono singoli performer che si sfidano ma gruppi-performer. Le sfide istituzionalizzate spesso non sono per niente scherzose, di ben altro tipo che quelle carnevalesche (contemporanee). Sono sfide che si esprimono in un'atmosfera aggressiva, antagonista, piena di tensione. Che nascondono a mala pena una rivalità pronta a passare a vie di fatto. A fuoriuscite dalla festa. Gli universi festivi sono svariati e complessi. Le tensioni, rivalità, contrapposizioni tra individui e gruppi sono espressione di processi storici e assetti sociali straordinariamente diversi, in giro per il mondo. Ma la festa come arena di sfide interne alle società è una costante transculturale» (Apolito 2014: 242).

\section{RIASSUNTI}

La questua di denaro, di alimenti e di capi di bestiame, il sacrificio di questi ultimi e il banchetto collettivo sono ancora oggi fra le azioni rituali più importanti delle feste campestri della Sardegna. Attraverso la descrizione e l'analisi di alcuni casi di studio, il contributo si propone di indagare l'importanza del cibo in ambito festivo. Durante il banchetto, infatti, si creano e si rinnovano relazioni e gerarchie, si esalta l'abbondanza e il consumo, si ribadisce l'appartenenza alla comunità e al culto comunitario. Il cibo, in sostanza, diviene un importante e imprescindibile strumento di comunicazione sociale.

The begging of money, food and livestock, the sacrifice of the latter and the collective banquet are still today among the most important ritual actions of the Sardinian rural festivals. Through the description and analysis of some case studies, the contribution aims to investigate the importance of food in the festive sphere. In fact, during the banquet, relations and hierarchies are created and renewed, abundance and consumption are exalted, membership of community and community worship is reiterated. In essence, food becomes an important and inescapable tool for social communication.

\section{INDICE}

Keywords : rural festivals, begging, sacrifice, ritual banquet, Sardinia

Parole chiave : feste campestri, questua, sacrificio, banchetto rituale, Sardegna

\section{AUTORE}

\section{SEBASTIANO MANNIA}

Università degli Studi di Palermo sebastiano.mannia[at]unipa.it 